\title{
Reviewers 2008 (Correction)
}

\author{
(C) ISS 2009
}

\section{Skeletal Radiol (2009) 38:105}

The text above the Reviewers should read as follows:

The editors of Skeletal Radiology would like to use this opportunity with the first issue of the New Year to thank our reviewers and members of the editorial board for their invaluable services and contributions to the journal. Reviewers, anonymous and hidden from view, play a huge part in influencing the quality of a journal as well as in the expeditious treatment of manuscripts. The support that the editors of Skeletal Radiology received from its reviewers in 2008 was nothing short of extraordinary. The average time taken to perform a review was under 14 days. The average time taken to repond to an invitation to review was under four days. Eighty percent of invitations to review were accepted right away. Not measurable or quantifiable is the high quality of the reviews. The time and intellectual rigor invested by our reviewers in refining and improving a manuscript not only eases the tasks of the editors but gives us a privileged position of witnessing skill, objectivity, fairness and experience, all coming together, in a productive manner. The editors most gratefully thank our reviewers for their support of the Journal and the high quality of their contributions.

The Reviewers 2008 can be found at doi:10.1007/s00256-008-0610-9. 